OPEN ACCESS

Edited by:

Gengyuan Liu,

Beijing Normal University, China

Reviewed by:

Gulnihal Ozbay,

Delaware State University,

United States

Tanvir Ahmed,

Bangladesh University of Engineering

and Technology, Bangladesh

${ }^{*}$ Correspondence:

Mahmood Yaseen Hachim

mahmood.almashhadani@mbru.ac.ae

Suad Hannawi

suad1@ausdoctors.net

tThese authors have contributed equally to this work

Specialty section: This article was submitted to Environmental health and Exposome,

a section of the journal

Frontiers in Public Health

Received: 18 October 2020

Accepted: 03 February 2021

Published: 19 March 2021

Citation:

Hachim MY, Hachim IY, Naeem K, Hannawi H, Al Salmi I and Hannawi S (2021) Higher Temperatures, Higher Solar Radiation, and Less Humidity Is

Associated With Poor Clinical and

Laboratory Outcomes in COVID-19

Patients.

Front. Public Health 9:618828.

doi: 10.3389/fpubh.2021.618828

\section{Higher Temperatures, Higher Solar Radiation, and Less Humidity Is Associated With Poor Clinical and Laboratory Outcomes in COVID-19 Patients}

\author{
Mahmood Yaseen Hachim ${ }^{1 * t}$, Ibrahim Y. Hachim ${ }^{2 \dagger}$, Kashif Naeem ${ }^{3 \dagger}$, Haifa Hannawi ${ }^{3 \dagger}$, \\ Issa Al Salmi ${ }^{4+}$ and Suad Hannawi ${ }^{3 *+}$
}

${ }^{1}$ Mohammed Bin Rashid University of Medicine and Health Sciences, Dubai, United Arab Emirates, ${ }^{2}$ College of Medicine, University of Sharjah, Sharjah, United Arab Emirates, ${ }^{3}$ Ministry of Health and Prevention (United Arab Emirates), Dubai, United Arab Emirates, ${ }^{4}$ The Royal Hospital, Muscat, Oman

Background: The COVID-19 pandemic varies between countries, with suggestions that weather might contribute to the transmission mode, disease presentation, severity, and clinical outcomes. Yet the exact link between climate and COVID-19 is still not well-explored.

Objectives: This study aimed to evaluate the effect of hot geographical region weather [like United Arab Emirates (UAE)] on COVID-19 clinical profile and outcomes. Temperature, wind speed, cloud cover, precipitation, and other weather-related variables were studied concerning COVID-19 patients outcomes and laboratory results.

Methodology: A total of 434 COVID-19 positive patients admitted between January and June 2020, were recruited from Al Kuwait Hospital, Dubai, UAE. Temperature, wind speed, cloud cover, and precipitation rate were retrieved from history+ for the day when COVID-19 patients presented to the hospital. These weather parameters were correlated with COVID-19 clinical and laboratory parameters.

Results: Our results showed that patients needed admission in days with higher temperatures, higher solar radiation, and less humidity were associated with higher deaths. This association can be linked to the association of these weather parameters with age at diagnosis; higher C-reactive protein (CRP), neutrophil count, white cell count (WCC), aspartate aminotransferase (AST), and alkaline phosphatase (ALP); and lower lymphocyte count, estimated glomerular filtration rate (eGFR), hemoglobin ( $\mathrm{Hb}), \mathrm{Na}$, and albumin, all of which are considered poor prognostic factors for COVID-19.

Conclusion: Our study highlighted the importance of weather-related variables on the dynamics of mortality and clinical outcomes of COVID-19. The hot weather might makes some people, especially those with comorbidities or older ages, develop aggressive inflammation that ends up with complications and mortality.

Keywords: COVID-19, SARS-CoV-2, environmental, weather, pandemic (COVID-19) 


\section{INTRODUCTION}

The exact link between weather and COVID-19 spread is still not well-explored, although a few reports claimed that warm weather can slow down such spread and can help in predicting which geographic areas in different countries can have a higher risk of spread (1). One of the COVID-19 pandemic characteristics is a very rapid spread and high mortality rates in countries north of the equator known to have low seasonal air temperatures (2). Such countries with low humidity are suspected to favor the transmission and survival of SARS-COV-2 (3). Such a link is not surprising for the virus family as Middle East respiratory syndrome coronavirus (MERS-CoV) human cases in Saudi Arabia were more likely to occur when conditions were relatively cold and dry (4), where increasing temperature to $65^{\circ} \mathrm{C}$ had a strong negative effect on viral infectivity (5). Recently, the severity of COVID-19 in Europe was documented to be decreased significantly between March and May, and the seasonality of COVID-19 was suggested to explain that note (6).

Some reports suggested SARS-CoV-2 be inactivated relatively fast during summer due to the sunlight effect (7), and the overall epidemic intensity of COVID-19 was shown to be reduced slightly following days with higher temperatures (8). Short-term exposures to the ozone can influence COVID-19 transmission and initiation of the disease (9).
The COVID-19 pandemic was found to be correlated negatively with average temperature (10), wind speed 14 days ago, the temperature of the day (11), air quality (12) in terms of averaged ground levels of particulate matter concentrations (13), and relative humidity (14). Of these, temperature and humidity are essential features for predicting the COVID-19 mortality rate (15). Air pollution by an increase in $\mathrm{PM}_{2.5}$ accelerated transmission of SARS-CoV-2 (16) and triggered COVID-19 spread and lethality levels (17).

Nevertheless, cases in warm and humid countries have consistently increased later, opposite to the claimed effect of warm weather on the virus spread (18). On the other hand, some reports showed that there was no association between COVID-19 transmission and temperature or UV radiation in Chinese cities (19). For example, the temperature was shown to have no role in the containment of COVID-19 in Wuhan (20).

From the literature, such as dynamic multidimensional and complex weather, COVID-19 interaction cannot be explained as a general role. Still, they can suggest a regional trend that should be kept in mind when trying to understand pandemic dynamics. Based on that, we thought of exploring the correlation between weather parameters in Dubai, United Arab Emirates (UAE), and COVID-19 patients' related clinical and laboratory characteristics. To our knowledge, this paper is the first to explore this relationship in the Middle East region.

TABLE 1 | The temperature, wind speed, cloud cover, precipitation rate, and other weather parameters of Dubai City for the duration of patient recruitment using history+ (https://www.meteoblue.com/en/historyplus).

\begin{tabular}{|c|c|c|c|c|c|}
\hline Timestamp & Variable & Unit & Level & Resolution & Aggregation \\
\hline Temperature [2 m elevation corrected] & Temperature & ${ }^{\circ} \mathrm{C}$ & $2 \mathrm{~m}$ elevation corrected & Daily & Minimum \\
\hline Temperature [2 m elevation corrected] & Temperature & ${ }^{\circ} \mathrm{C}$ & $2 \mathrm{~m}$ elevation corrected & Daily & Maximum \\
\hline Temperature [2 m elevation corrected] & Temperature & ${ }^{\circ} \mathrm{C}$ & $2 \mathrm{~m}$ elevation corrected & Daily & Mean \\
\hline Relative humidity [2 m] & Relative humidity & $\%$ & $2 \mathrm{~m}$ & Daily & Minimum \\
\hline Relative humidity [2 m] & Relative humidity & $\%$ & $2 \mathrm{~m}$ & Daily & Maximum \\
\hline Relative humidity [2 m] & Relative humidity & $\%$ & $2 \mathrm{~m}$ & Daily & Mean \\
\hline Precipitation total & Precipitation total, mm & $\mathrm{mm}$ & sfc & Daily & Summation \\
\hline Cloud Cover Total & Cloud cover total & $\%$ & sfc & Daily & Mean \\
\hline Sunshine duration & Sunshine duration & $\min$ & sfc & Daily & Summation \\
\hline Shortwave radiation & Shortwave radiation & $\mathrm{W} / \mathrm{m}^{2}$ & sfc & Daily & Summation \\
\hline Direct shortwave radiation & Direct shortwave radiation & $\mathrm{W} / \mathrm{m}^{2}$ & sfc & Daily & Summation \\
\hline Evapotranspiration & Evapotranspiration & $\mathrm{mm}$ & sfc & Daily & Summation \\
\hline Wind speed [10 m] & Wind speed & $\mathrm{km} / \mathrm{h}$ & $10 \mathrm{~m}$ & Daily & Minimum \\
\hline Wind speed [10 m] & Wind speed & $\mathrm{km} / \mathrm{h}$ & $10 \mathrm{~m}$ & Daily & Maximum \\
\hline Wind speed [10 m] & Wind speed & $\mathrm{km} / \mathrm{h}$ & $10 \mathrm{~m}$ & Daily & Mean \\
\hline Wind direction dominant [10 m] & Wind direction dominant & $\circ$ & $10 \mathrm{~m}$ & Daily & None \\
\hline Temperature [1,000 mb] & Temperature & ${ }^{\circ} \mathrm{C}$ & $1,000 \mathrm{mb}$ & Daily & Minimum \\
\hline Temperature $[1,000 \mathrm{mb}]$ & Temperature & ${ }^{\circ} \mathrm{C}$ & $1,000 \mathrm{mb}$ & Daily & Maximum \\
\hline Temperature $[1,000 \mathrm{mb}]$ & Temperature & ${ }^{\circ} \mathrm{C}$ & $1,000 \mathrm{mb}$ & Daily & Mean \\
\hline Temperature & Temperature & ${ }^{\circ} \mathrm{C}$ & sfc & Daily & Minimum \\
\hline Temperature & Temperature & ${ }^{\circ} \mathrm{C}$ & sfc & Daily & Maximum \\
\hline Temperature & Temperature & ${ }^{\circ} \mathrm{C}$ & sfc & Daily & Mean \\
\hline
\end{tabular}

${ }^{\circ} \mathrm{C}$, degrees Celsius; $\mathrm{W} / \mathrm{m}^{2}$, watt per square meter; $\mathrm{km} / \mathrm{h}$, kilometer per hour; $\mathrm{mm}$, millimeters; sfc, surface temperature. 
TABLE 2 | Difference in day-of-admission weather variables in Dubai City between COVID-19 patients' clinical outcomes divided into those with risk factors to develop severe COVID-19 and those without (A: old age, B: DM, C: HTN, D: CVD, or E: chronic lung disease).

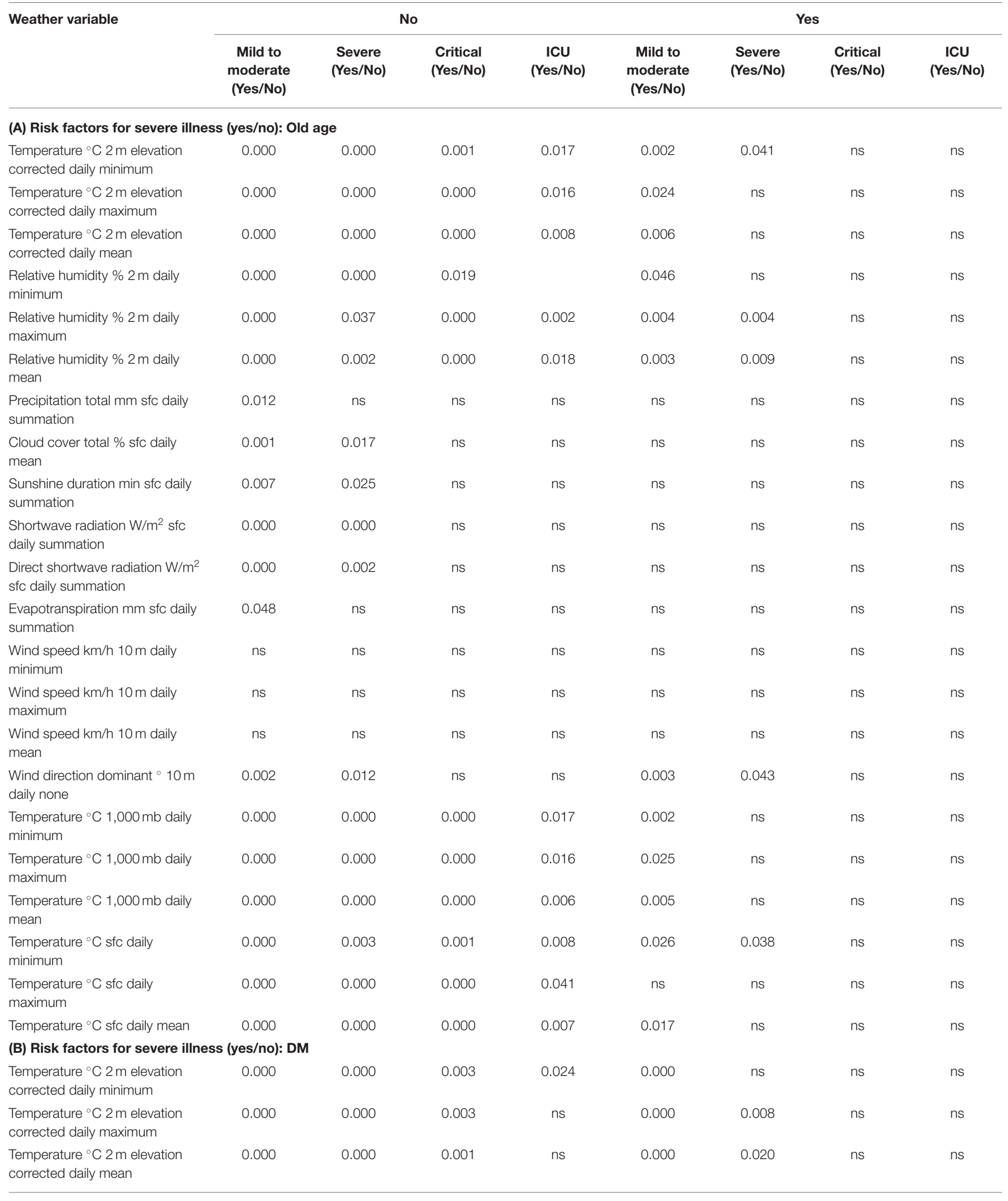


TABLE 2 | Continued

\begin{tabular}{|c|c|c|c|c|c|c|c|c|}
\hline Weather variable & \multicolumn{4}{|c|}{ No } & \multicolumn{4}{|c|}{ Yes } \\
\hline $\begin{array}{l}\text { Relative humidity } \% 2 \text { m daily } \\
\text { minimum }\end{array}$ & 0.000 & 0.000 & ns & ns & 0.002 & 0.011 & 0.012 & ns \\
\hline $\begin{array}{l}\text { Relative humidity } \% 2 \text { m daily } \\
\text { mean }\end{array}$ & 0.000 & 0.000 & 0.006 & ns & 0.000 & ns & ns & ns \\
\hline $\begin{array}{l}\text { Precipitation total } \mathrm{mm} \text { sfc daily } \\
\text { summation }\end{array}$ & 0.021 & ns & ns & ns & ns & ns & ns & ns \\
\hline $\begin{array}{l}\text { Cloud cover total \% sfc daily } \\
\text { mean }\end{array}$ & 0.015 & ns & ns & ns & ns & ns & ns & ns \\
\hline $\begin{array}{l}\text { Direct shortwave radiation } \mathrm{W} / \mathrm{m}^{2} \\
\text { sfc daily summation }\end{array}$ & 0.000 & 0.008 & ns & ns & ns & ns & ns & ns \\
\hline $\begin{array}{l}\text { Evapotranspiration mm sfc daily } \\
\text { summation }\end{array}$ & ns & ns & ns & ns & ns & ns & ns & ns \\
\hline $\begin{array}{l}\text { Wind speed } \mathrm{km} / \mathrm{h} 10 \mathrm{~m} \text { daily } \\
\text { minimum }\end{array}$ & ns & ns & ns & ns & 0.036 & ns & ns & ns \\
\hline $\begin{array}{l}\text { Wind speed } \mathrm{km} / \mathrm{h} 10 \mathrm{~m} \text { daily } \\
\text { maximum }\end{array}$ & ns & ns & ns & ns & ns & ns & ns & ns \\
\hline $\begin{array}{l}\text { Wind speed km/h } 10 \text { m daily } \\
\text { mean }\end{array}$ & ns & ns & ns & ns & ns & ns & ns & ns \\
\hline $\begin{array}{l}\text { Wind direction dominant }{ }^{\circ} 10 \mathrm{~m} \\
\text { daily none }\end{array}$ & 0.000 & 0.007 & ns & 0.028 & ns & ns & ns & ns \\
\hline $\begin{array}{l}\text { Temperature }{ }^{\circ} \mathrm{C} \text { sfc daily } \\
\text { maximum }\end{array}$ & 0.000 & 0.000 & 0.009 & ns & 0.000 & 0.013 & ns & ns \\
\hline Temperature ${ }^{\circ} \mathrm{C}$ sfc daily mean & 0.000 & 0.000 & 0.001 & 0.048 & 0.000 & 0.023 & ns & ns \\
\hline \multicolumn{9}{|c|}{ (C) Risk factors for severe illness (yes/no): HTN } \\
\hline $\begin{array}{l}\text { Temperature }{ }^{\circ} \mathrm{C} 2 \mathrm{~m} \text { elevation } \\
\text { corrected daily minimum }\end{array}$ & 0.000 & 0.000 & 0.001 & 0.050 & 0.000 & ns & ns & ns \\
\hline $\begin{array}{l}\text { Temperature }{ }^{\circ} \mathrm{C} 2 \mathrm{~m} \text { elevation } \\
\text { corrected daily maximum }\end{array}$ & 0.000 & 0.000 & 0.000 & 0.024 & 0.001 & ns & ns & ns \\
\hline $\begin{array}{l}\text { Temperature }{ }^{\circ} \mathrm{C} 2 \mathrm{~m} \text { elevation } \\
\text { corrected daily mean }\end{array}$ & 0.000 & 0.000 & 0.000 & 0.016 & 0.000 & ns & ns & ns \\
\hline $\begin{array}{l}\text { Relative humidity } \% 2 \text { m daily } \\
\text { minimum }\end{array}$ & 0.000 & 0.000 & 0.012 & & & ns & ns & ns \\
\hline $\begin{array}{l}\text { Relative humidity } \% 2 \text { m daily } \\
\text { maximum }\end{array}$ & 0.000 & 0.016 & 0.000 & 0.001 & 0.003 & ns & ns & ns \\
\hline $\begin{array}{l}\text { Relative humidity } \% 2 \text { m daily } \\
\text { mean }\end{array}$ & 0.000 & 0.000 & 0.000 & 0.003 & 0.013 & ns & ns & ns \\
\hline $\begin{array}{l}\text { Precipitation total mm sfc daily } \\
\text { summation }\end{array}$ & 0.012 & ns & ns & ns & ns & ns & ns & ns \\
\hline
\end{tabular}


TABLE 2 | Continued

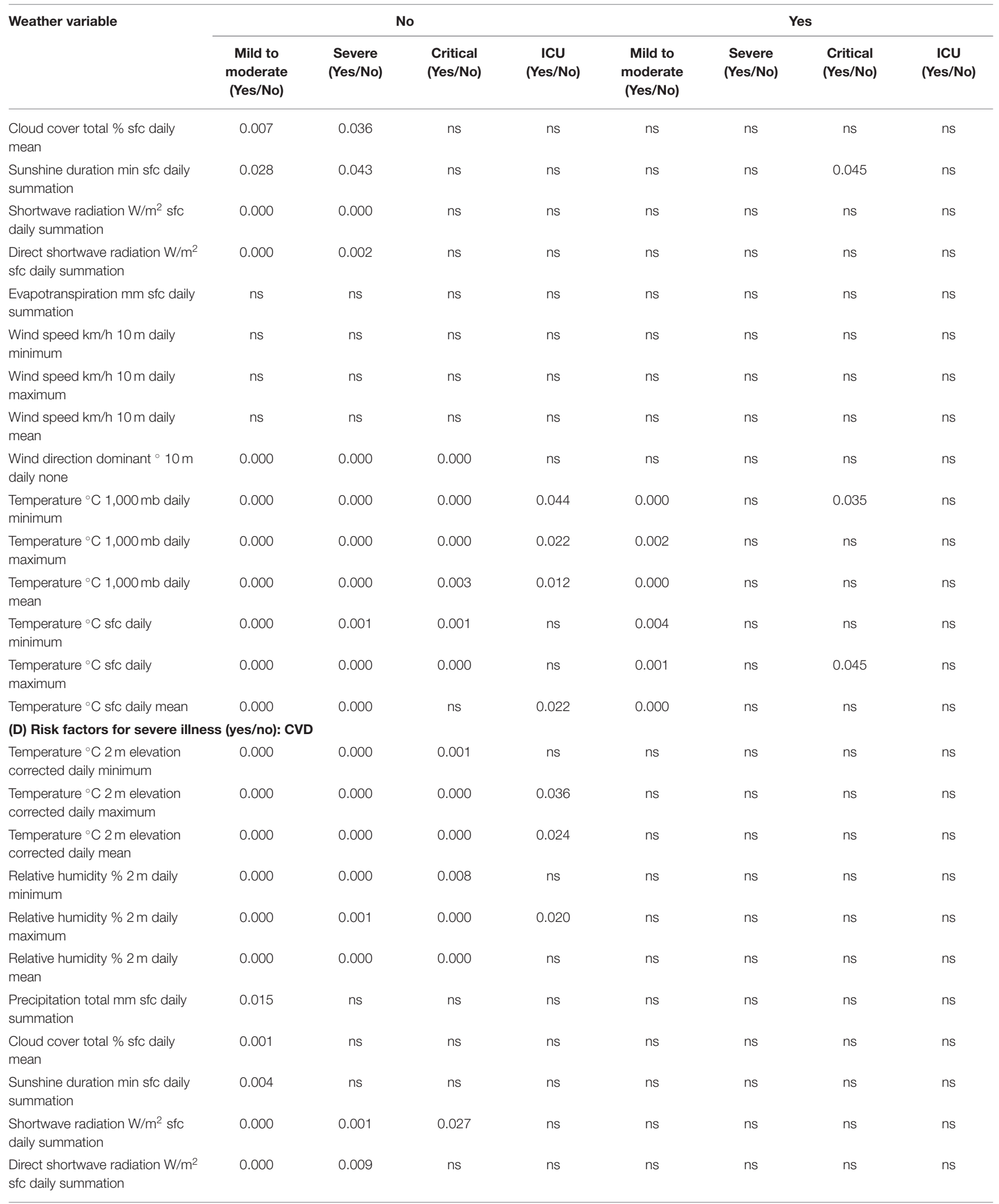


TABLE 2 | Continued

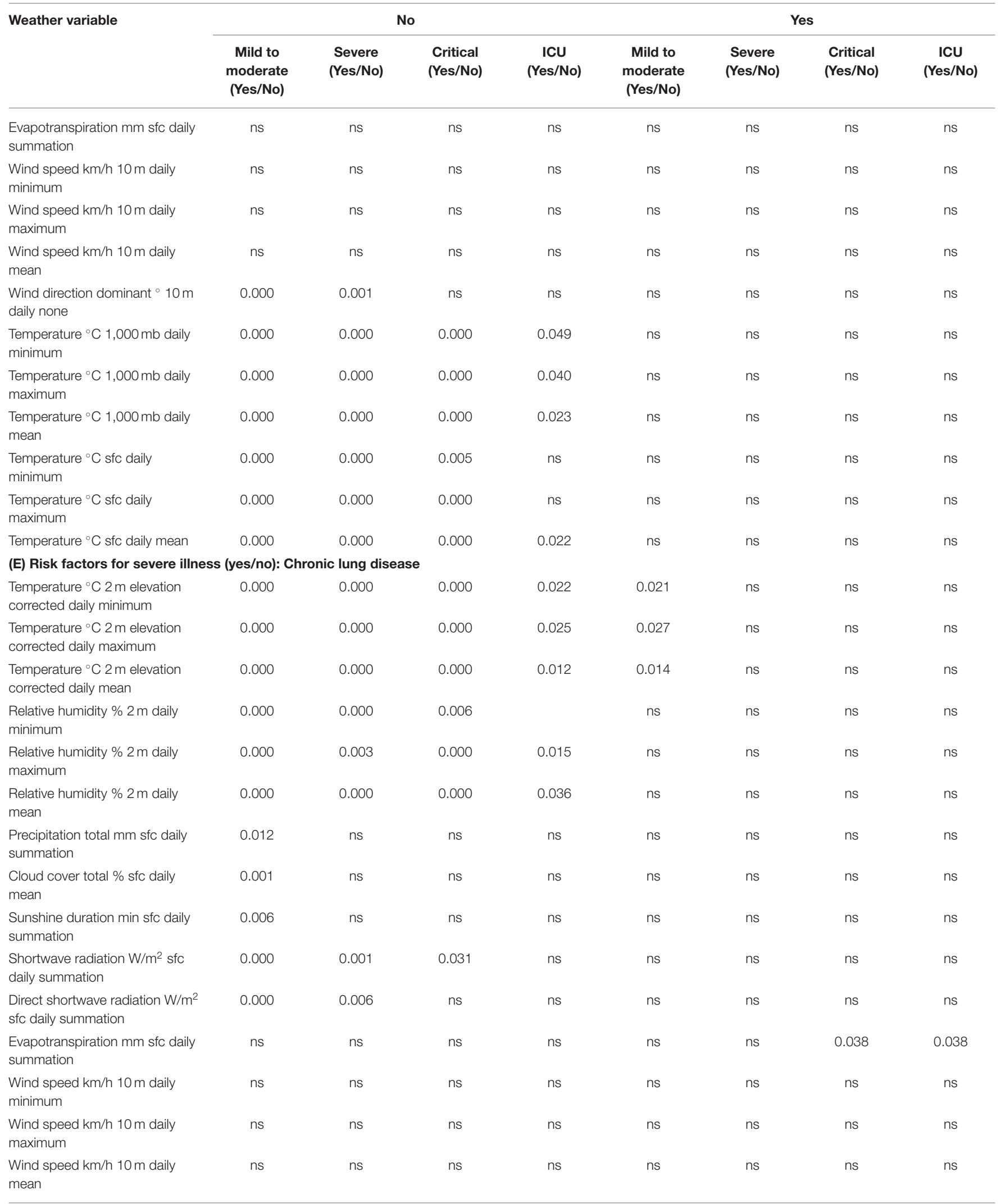


TABLE 2 | Continued

\begin{tabular}{|c|c|c|c|c|c|c|c|c|}
\hline \multirow[t]{2}{*}{ Weather variable } & \multicolumn{4}{|c|}{ No } & \multicolumn{4}{|c|}{ Yes } \\
\hline & $\begin{array}{c}\text { Mild to } \\
\text { moderate } \\
\text { (Yes/No) }\end{array}$ & $\begin{array}{l}\text { Severe } \\
\text { (Yes/No) }\end{array}$ & $\begin{array}{l}\text { Critical } \\
\text { (Yes/No) }\end{array}$ & $\begin{array}{c}\text { ICU } \\
\text { (Yes/No) }\end{array}$ & $\begin{array}{c}\text { Mild to } \\
\text { moderate } \\
\text { (Yes/No) }\end{array}$ & $\begin{array}{l}\text { Severe } \\
\text { (Yes/No) }\end{array}$ & $\begin{array}{l}\text { Critical } \\
\text { (Yes/No) }\end{array}$ & $\begin{array}{c}\text { ICU } \\
\text { (Yes/No) }\end{array}$ \\
\hline $\begin{array}{l}\text { Wind direction dominant }{ }^{\circ} 10 \mathrm{~m} \\
\text { daily none }\end{array}$ & 0.000 & 0.001 & & & $\mathrm{~ns}$ & ns & ns & ns \\
\hline $\begin{array}{l}\text { Temperature }{ }^{\circ} \mathrm{C} 1,000 \mathrm{mb} \text { daily } \\
\text { minimum }\end{array}$ & 0.000 & 0.000 & 0.000 & 0.026 & 0.029 & ns & ns & ns \\
\hline $\begin{array}{l}\text { Temperature }{ }^{\circ} \mathrm{C} 1,000 \mathrm{mb} \text { daily } \\
\text { maximum }\end{array}$ & 0.000 & 0.000 & 0.000 & 0.027 & 0.021 & ns & ns & ns \\
\hline $\begin{array}{l}\text { Temperature }{ }^{\circ} \mathrm{C} 1,000 \mathrm{mb} \text { daily } \\
\text { mean }\end{array}$ & 0.000 & 0.000 & 0.000 & 0.012 & 0.018 & ns & ns & ns \\
\hline $\begin{array}{l}\text { Temperature }{ }^{\circ} \mathrm{C} \text { sfc daily } \\
\text { minimum }\end{array}$ & 0.000 & 0.001 & 0.003 & 0.030 & ns & ns & ns & ns \\
\hline $\begin{array}{l}\text { Temperature }{ }^{\circ} \mathrm{C} \text { sfc daily } \\
\text { maximum }\end{array}$ & 0.000 & 0.000 & 0.000 & 0.049 & ns & ns & ns & ns \\
\hline Temperature ${ }^{\circ} \mathrm{C}$ sfc daily mean & 0.000 & 0.000 & 0.000 & 0.012 & 0.016 & ns & ns & ns \\
\hline
\end{tabular}

\section{MATERIALS AND METHODS}

\section{Patient Data Collection}

A total of 434 COVID-19-positive patients admitted between January and June 2020 were recruited from Al Kuwait Hospital, Dubai, UAE. The study was approved by the Ministry of Health and Prevention (MOHAP, Research Ethics Committee number MOHAP/DXB-REC/MMM/NO. 44/2020). Adult patients (above 18 years) with COVID-19 (confirmed by nasopharyngeal polymerase chain reaction, PCR-positive sample) were enrolled. Complete current and past medical history, along with their demographic data, history of recent travel or contact with another confirmed case(s), was documented. Patients were classified according to "Clinical Management of Critically Ill COVID-19 Patients" guidelines (Version 1, April 15, 2020) issued by MOHAP (6).

\section{Blood and Radiological Tests}

Laboratory tests were retrieved: (1) complete blood count, including neutrophil count (NR: $\left.2-7 \times 10^{3} / \mu \mathrm{L}\right)$, lymphocyte count (NR: $\left.1-3 \times 10^{3} / \mu \mathrm{L}\right)$, hemoglobin (Hb, NR: 12-15 $\mathrm{g} / \mathrm{dL}$ ), white cell count (WCC, NR: $4-11 \times 10^{3} / \mu \mathrm{L}$ ), and platelet count (NR: $\left.150-450 \times 10^{3} / \mu \mathrm{L}\right)$; (2) coagulation profile, including international normalized ratio (INR, NR: 0.8-1.29 s), prothrombin time (PT, NR: 9.9-12.3 s); (3) electrolytes, including sodium (Na, NR: 136-145 mmol/L) and potassium (K, NR: 3.6$5.1 \mathrm{mmol} / \mathrm{L}$ ); (4) renal function tests, including urea (NR: 2.5$6.5 \mathrm{mmol} / \mathrm{L}$ ), creatinine (NR: $53-88 \mu \mathrm{mol} / \mathrm{L}$ ), and estimated glomerular filtration rate (eGFR, NR: $90-120 \mathrm{~mL} / \mathrm{min} / 1.73$ $\mathrm{m}^{2}$ ); (5) liver function tests, including total serum bilirubin (NR: 3-17 $\mu \mathrm{mol} / \mathrm{L}$ ), alanine aminotransferase (ALT, NR: 16$63 \mathrm{IU} / \mathrm{L}$ ), aspartate aminotransferase (AST, NR: 15-37 U/L), alkaline phosphatase (ALP, NR: 46-116 IU/L), and albumin (NR: $34-50 \mathrm{~g} / \mathrm{L}$ ); (6) inflammatory markers, including C-reactive protein (CRP, NR: 0-3 mg/L), D-dimers (NR: $\mathrm{mg} / \mathrm{dL}$ ), lactate dehydrogenase (LDH, NR: 85-227 IU/L), procalcitonin (NR: $\mu \mathrm{g} / \mathrm{L})$, and ferritin $(8-388 \mu \mathrm{g} / \mathrm{L})$. For risk of severe cases, the presence of lymphopenia, neutrophilia, high ALT and/or AST, high LDH, high CRP, high ferritin, high D-dimer, and high pro-calcitonin, above those of the age- and gender-matched references, were used as indicators of risk. Admission chest Xray (presence of bilateral air consolidation) and computerized tomography (CT) scan (presence of bilateral peripheral groundglass opacities) were documented.

\section{Climate Data}

We downloaded the temperature, wind speed, cloud cover, precipitation rate, and other weather parameters of Dubai City for the duration of patient recruitment using history+ (https:// www.meteoblue.com/en/historyplus), which offers immediate access to the meteoblue global weather simulation archive as shown in Table 1. We matched the date of admission for each patient with the corresponding day weather details, as shown in Table 1.

\section{Statistical Analysis}

For all statistical analyses and tests, SPSS was used (IBM SPSS Statistics for Windows, Version 26.0, released 2019, IBM Corp., Armonk, NY). The chi-square test of independence was used to examine the association between categorical variables. Pearson's correlation coefficient was used to measure the correlation between different variables where correlation is significant at the 0.01 and 0.05 levels (two-tailed).

\section{RESULTS}

\section{The Clinical Severity of COVID-19 Was Significantly Dependent on the Temperature on the Day of Admission}

Comparing the weather parameters on the day of admission between patients with different COVID-19 severity levels or those who needed ICU admission showed that daily temperature 
TABLE 3A | Difference in day-of-admission weather variables in Dubai City between COVID-19 patients' clinical outcomes: Death.

\begin{tabular}{|c|c|c|c|c|c|}
\hline \multirow[t]{2}{*}{ Weather variable } & \multicolumn{4}{|c|}{ Death } & \multirow[t]{2}{*}{$p$-value } \\
\hline & Mean & SD & Mean & SD & \\
\hline Temperature ${ }^{\circ} \mathrm{C} 2 \mathrm{~m}$ elevation corrected daily minimum & 22.24 & 4.22 & 23.09 & 3.50 & ns \\
\hline Temperature ${ }^{\circ} \mathrm{C} 2 \mathrm{~m}$ elevation corrected daily maximum & 37 & 5.92 & 38.8 & 4.79 & 0.016 \\
\hline Temperature ${ }^{\circ} \mathrm{C} 2 \mathrm{~m}$ elevation corrected daily mean & 29.01 & 4.89 & 30.46 & 3.98 & 0.02 \\
\hline Relative humidity $\% 2$ m daily maximum & 72 & 18 & 66 & 18 & 0.012 \\
\hline Relative humidity $\% 2 \mathrm{~m}$ daily mean & 45.31 & 14.17 & 40.15 & 13.93 & 0.005 \\
\hline Precipitation total mm sfc daily summation & 0.305 & 1.60 & 0.11 & 0.35 & ns \\
\hline Cloud cover total \% sfc daily mean & 22.29 & 25.77 & 16.12 & 22.08 & ns \\
\hline Sunshine duration min sfc daily summation & 615.2 & 199.96 & 657.4 & 165.95 & ns \\
\hline Shortwave radiation $\mathrm{W} / \mathrm{m}^{2}$ sfc daily summation & 6,933 & 1266.91 & 7296 & 954.79 & 0.023 \\
\hline Wind speed $\mathrm{km} / \mathrm{h} 10 \mathrm{~m}$ daily mean & 8.312 & 3.30 & 8.471 & 3.78 & ns \\
\hline Wind direction dominant ${ }^{\circ} 10 \mathrm{~m}$ daily none & 196.1 & 114.62 & 189.5 & 106.88 & ns \\
\hline Temperature ${ }^{\circ} \mathrm{C} 1,000 \mathrm{mb}$ daily minimum & 24.17 & 5.73 & 25.65 & 5.02 & 0.043 \\
\hline Temperature ${ }^{\circ} \mathrm{C} 1,000 \mathrm{mb}$ daily maximum & 34.72 & 5.77 & 36.45 & 4.77 & 0.018 \\
\hline Temperature ${ }^{\circ} \mathrm{C} 1,000 \mathrm{mb}$ daily mean & 29.08 & 5.68 & 30.85 & 4.80 & 0.014 \\
\hline Temperature ${ }^{\circ} \mathrm{C}$ sfc daily minimum & 19.9 & 4.01 & 20.54 & 3.65 & ns \\
\hline Temperature ${ }^{\circ} \mathrm{C}$ sfc daily maximum & 44.81 & 6.76 & 46.71 & 5.34 & 0.026 \\
\hline Temperature ${ }^{\circ} \mathrm{C}$ sfc daily mean & 30.71 & 4.91 & 32.12 & 3.97 & 0.024 \\
\hline
\end{tabular}

TABLE 3B | Difference in day-of-admission weather variables in Dubai City between COVID-19 patients' clinical outcomes: Acute cardiac injury.

\begin{tabular}{|c|c|c|c|c|c|}
\hline \multirow[t]{3}{*}{ Weather variable } & \multicolumn{4}{|c|}{ Acute cardiac injury } & \multirow[t]{3}{*}{$p$-value } \\
\hline & \multicolumn{2}{|c|}{ No } & \multicolumn{2}{|c|}{ Yes } & \\
\hline & Mean & SD & Mean & SD & \\
\hline Temperature ${ }^{\circ} \mathrm{C} 2 \mathrm{~m}$ elevation corrected daily minimum & 22.08 & 4.24 & 23.43 & 3.49 & 0.004 \\
\hline Temperature ${ }^{\circ} \mathrm{C} 2 \mathrm{~m}$ elevation corrected daily maximum & 36.75 & 5.96 & 39.17 & 4.73 & 0 \\
\hline Temperature ${ }^{\circ} \mathrm{C} 2 \mathrm{~m}$ elevation corrected daily mean & 28.81 & 4.93 & 30.77 & 3.89 & 0 \\
\hline Relative humidity $\% 2 \mathrm{~m}$ daily mean & 45.19 & 14.53 & 41.94 & 12.99 & 0.048 \\
\hline Precipitation total $\mathrm{mm}$ sfc daily summation & 0.32 & 1.66 & 0.10 & 0.36 & ns \\
\hline Cloud cover total \% sfc daily mean & 22.39 & 25.78 & 17.45 & 23.22 & ns \\
\hline Sunshine duration min sfc daily summation & 614.48 & 197.32 & 648.59 & 186.44 & ns \\
\hline Shortwave radiation $\mathrm{W} / \mathrm{m}^{2}$ sfc daily summation & 6903.76 & 1268.17 & 7298.58 & 1023.49 & 0.005 \\
\hline Wind speed km/h $10 \mathrm{~m}$ daily mean & 8.31 & 3.34 & 8.45 & 3.56 & ns \\
\hline Wind direction dominant ${ }^{\circ} 10 \mathrm{~m}$ daily none & 194.25 & 115.45 & 197.88 & 106.02 & ns \\
\hline Temperature ${ }^{\circ} \mathrm{C} 1,000 \mathrm{mb}$ daily minimum & 23.98 & 5.77 & 25.90 & 4.90 & 0.003 \\
\hline Temperature ${ }^{\circ} \mathrm{C} 1,000 \mathrm{mb}$ daily maximum & 34.49 & 5.80 & 36.79 & 4.70 & 0 \\
\hline Temperature ${ }^{\circ} \mathrm{C} 1,000 \mathrm{mb}$ daily mean & 28.87 & 5.74 & 31.09 & 4.64 & 0.001 \\
\hline Temperature ${ }^{\circ} \mathrm{C}$ sfc daily minimum & 19.74 & 4.05 & 20.93 & 3.46 & 0.008 \\
\hline Temperature ${ }^{\circ} \mathrm{C}$ sfc daily maximum & 44.50 & 6.74 & 47.26 & 5.53 & 0 \\
\hline Temperature ${ }^{\circ} \mathrm{C}$ sfc daily mean & 30.48 & 4.93 & 32.55 & 3.94 & 0 \\
\hline
\end{tabular}


TABLE 3C | Difference in day-of-admission weather variables in Dubai City between COVID-19 patients' clinical outcomes: Acute kidney injury.

\begin{tabular}{|c|c|c|c|c|c|}
\hline \multirow[t]{2}{*}{ Weather variable } & \multicolumn{4}{|c|}{ Acute kidney injury } & \multirow[t]{2}{*}{$p$-value } \\
\hline & Mean & SD & Mean & SD & \\
\hline Temperature ${ }^{\circ} \mathrm{C} 2 \mathrm{~m}$ elevation corrected daily minimum & 22.18 & 4.23 & 23.20 & 3.57 & 0.038 \\
\hline Temperature ${ }^{\circ} \mathrm{C} 2 \mathrm{~m}$ elevation corrected daily maximum & 36.95 & 5.97 & 38.64 & 4.83 & 0.015 \\
\hline Temperature ${ }^{\circ} \mathrm{C} 2 \mathrm{~m}$ elevation corrected daily mean & 28.95 & 4.92 & 30.42 & 3.97 & 0.01 \\
\hline Relative humidity $\% 2$ m daily maximum & 72.00 & 18.00 & 67.00 & 18.00 & ns \\
\hline Relative humidity $\% 2 \mathrm{~m}$ daily mean & 45.11 & 14.37 & 41.90 & 13.52 & ns \\
\hline Precipitation total mm sfc daily summation & 0.31 & 1.64 & 0.14 & 0.42 & ns \\
\hline Cloud cover total \% sfc daily mean & 21.96 & 25.70 & 18.59 & 23.52 & ns \\
\hline Sunshine duration min sfc daily summation & 618.12 & 196.51 & 638.05 & 190.41 & ns \\
\hline Shortwave radiation $\mathrm{W} / \mathrm{m}^{2}$ sfc daily summation & 6944.16 & 1250.10 & 7183.31 & 1120.31 & ns \\
\hline Wind speed $\mathrm{km} / \mathrm{h} 10 \mathrm{~m}$ daily mean & 8.31 & 3.35 & 8.45 & 3.53 & ns \\
\hline Wind direction dominant ${ }^{\circ} 10 \mathrm{~m}$ daily none & 193.67 & 114.86 & 200.58 & 107.30 & ns \\
\hline Temperature ${ }^{\circ} \mathrm{C} 1,000 \mathrm{mb}$ daily minimum & 24.15 & 5.78 & 25.46 & 4.95 & ns \\
\hline Temperature ${ }^{\circ} \mathrm{C} 1,000 \mathrm{mb}$ daily maximum & 34.68 & 5.80 & 36.31 & 4.79 & 0.016 \\
\hline Temperature ${ }^{\circ} \mathrm{C} 1,000 \mathrm{mb}$ daily mean & 29.03 & 5.72 & 30.71 & 4.77 & 0.012 \\
\hline Temperature ${ }^{\circ} \mathrm{C}$ sfc daily minimum & 19.81 & 4.03 & 20.76 & 3.57 & 0.046 \\
\hline Temperature ${ }^{\circ} \mathrm{C}$ sfc daily maximum & 44.77 & 6.78 & 46.50 & 5.54 & 0.029 \\
\hline Temperature ${ }^{\circ} \mathrm{C}$ sfc daily mean & 30.64 & 4.95 & 32.13 & 3.96 & 0.01 \\
\hline
\end{tabular}

TABLE 3D | Difference in day-of-admission weather variables in Dubai City between COVID-19 patients' clinical outcomes: Acute liver injury.

\begin{tabular}{|c|c|c|c|c|c|}
\hline \multirow[t]{3}{*}{ Weather variable } & \multicolumn{4}{|c|}{ Acute liver injury } & \multirow[t]{3}{*}{$p$-value } \\
\hline & \multicolumn{2}{|c|}{ No } & \multicolumn{2}{|c|}{ Yes } & \\
\hline & Mean & SD & Mean & SD & \\
\hline Temperature ${ }^{\circ} \mathrm{C} 2 \mathrm{~m}$ elevation corrected daily minimum & 22.05 & 4.24 & 23.74 & 3.29 & 0.001 \\
\hline Temperature ${ }^{\circ} \mathrm{C} 2 \mathrm{~m}$ elevation corrected daily maximum & 36.90 & 5.92 & 38.93 & 4.93 & 0.004 \\
\hline Temperature ${ }^{\circ} \mathrm{C} 2 \mathrm{~m}$ elevation corrected daily mean & 28.88 & 4.90 & 30.78 & 3.89 & 0.001 \\
\hline Relative humidity $\% 2 \mathrm{~m}$ daily mean & 44.85 & 14.42 & 42.86 & 13.47 & ns \\
\hline Precipitation total mm sfc daily summation & 0.31 & 1.64 & 0.12 & 0.37 & ns \\
\hline Cloud cover total \% sfc daily mean & 21.19 & 24.52 & 21.67 & 28.41 & ns \\
\hline Sunshine duration min sfc daily summation & 623.31 & 188.12 & 617.18 & 223.62 & ns \\
\hline Shortwave radiation $\mathrm{W} / \mathrm{m}^{2}$ sfc daily summation & 6958.65 & 1199.60 & 7131.62 & 1336.45 & ns \\
\hline Wind speed km/h $10 \mathrm{~m}$ daily mean & 8.22 & 3.20 & 8.84 & 4.04 & ns \\
\hline Wind direction dominant ${ }^{\circ} 10 \mathrm{~m}$ daily none & 192.74 & 114.09 & 204.72 & 110.08 & ns \\
\hline Temperature ${ }^{\circ} \mathrm{C} 1,000 \mathrm{mb}$ daily minimum & 23.97 & 5.75 & 26.27 & 4.74 & 0.001 \\
\hline Temperature ${ }^{\circ} \mathrm{C} 1,000 \mathrm{mb}$ daily maximum & 34.64 & 5.76 & 36.55 & 4.90 & 0.005 \\
\hline Temperature ${ }^{\circ} \mathrm{C} 1,000 \mathrm{mb}$ daily mean & 28.96 & 5.71 & 31.07 & 4.67 & 0.002 \\
\hline Temperature ${ }^{\circ} \mathrm{C}$ sfc daily minimum & 19.71 & 4.02 & 21.25 & 3.45 & 0.001 \\
\hline Temperature ${ }^{\circ} \mathrm{C}$ sfc daily maximum & 44.71 & 6.67 & 46.82 & 5.94 & 0.008 \\
\hline Temperature ${ }^{\circ} \mathrm{C}$ sfc daily mean & 30.57 & 4.93 & 32.52 & 3.87 & 0.001 \\
\hline
\end{tabular}


TABLE 3E | Difference in day-of-admission weather variables in Dubai City between COVID-19 patients' clinical outcomes: Acidosis.

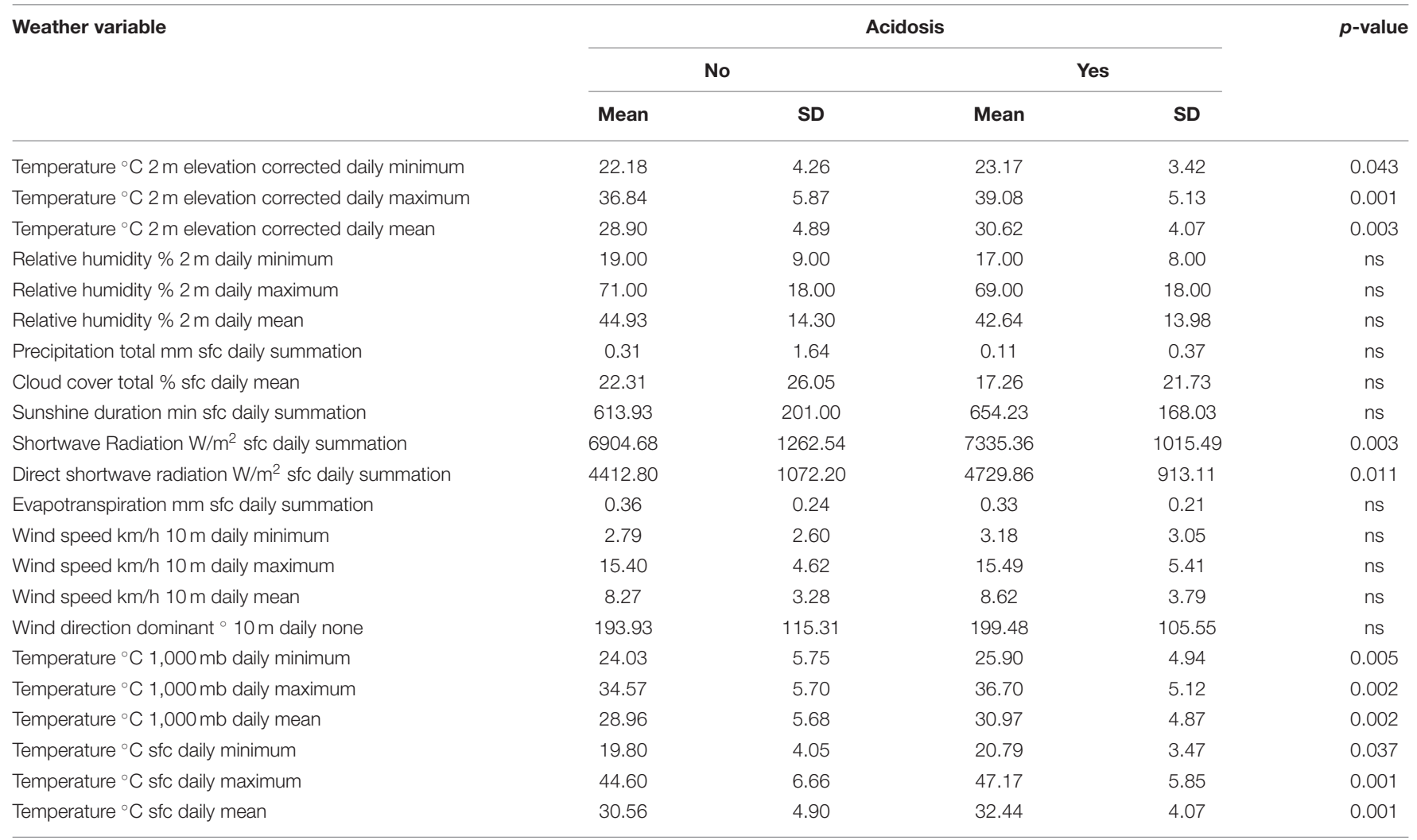

TABLE 3F I Difference in day-of-admission weather variables in Dubai City between COVID-19 patients' clinical outcomes: Septic shock.

\begin{tabular}{|c|c|c|c|c|c|}
\hline \multirow[t]{3}{*}{ Weather variable } & \multicolumn{4}{|c|}{ Septic Shock } & \multirow[t]{3}{*}{$p$-value } \\
\hline & \multicolumn{2}{|c|}{ No } & \multicolumn{2}{|c|}{ Yes } & \\
\hline & Mean & SD & Mean & SD & \\
\hline Temperature ${ }^{\circ} \mathrm{C} 2 \mathrm{~m}$ elevation corrected daily minimum & 22.20 & 4.20 & 23.34 & 3.58 & 0.033 \\
\hline Temperature ${ }^{\circ} \mathrm{C} 2 \mathrm{~m}$ elevation corrected daily maximum & 36.95 & 5.91 & 39.09 & 4.80 & 0.004 \\
\hline Temperature ${ }^{\circ} \mathrm{C} 2 \mathrm{~m}$ elevation corrected daily mean & 28.96 & 4.88 & 30.73 & 3.96 & 0.005 \\
\hline Relative humidity $\% 2 \mathrm{~m}$ daily mean & 45.20 & 14.32 & 40.63 & 13.32 & 0.014 \\
\hline Precipitation total mm sfc daily summation & 0.30 & 1.60 & 0.14 & 0.42 & ns \\
\hline Cloud cover total \% sfc daily mean & 22.44 & 25.84 & 15.27 & 21.35 & 0.03 \\
\hline Sunshine duration min sfc daily summation & 614.95 & 200.15 & 659.34 & 163.76 & ns \\
\hline Shortwave radiation $\mathrm{W} / \mathrm{m}^{2}$ sfc daily summation & 6933.76 & 1262.12 & 7295.28 & 983.52 & 0.024 \\
\hline Wind speed km/h $10 \mathrm{~m}$ daily mean & 8.28 & 3.28 & 8.65 & 3.92 & ns \\
\hline Wind direction dominant ${ }^{\circ} 10 \mathrm{~m}$ daily none & 195.87 & 114.88 & 190.85 & 105.41 & ns \\
\hline Temperature ${ }^{\circ} \mathrm{C} 1,000 \mathrm{mb}$ daily minimum & 24.09 & 5.71 & 26.10 & 4.98 & 0.006 \\
\hline Temperature ${ }^{\circ} \mathrm{C} 1,000 \mathrm{mb}$ daily maximum & 34.68 & 5.75 & 36.72 & 4.80 & 0.005 \\
\hline Temperature ${ }^{\circ} \mathrm{C} 1,000 \mathrm{mb}$ daily mean & 29.02 & 5.66 & 31.16 & 4.77 & 0.003 \\
\hline Temperature ${ }^{\circ} \mathrm{C}$ sfc daily minimum & 19.86 & 3.99 & 20.76 & 3.69 & ns \\
\hline Temperature ${ }^{\circ} \mathrm{C}$ sfc daily maximum & 44.75 & 6.72 & 47.05 & 5.43 & 0.007 \\
\hline Temperature ${ }^{\circ} \mathrm{C}$ sfc daily mean & 30.67 & 4.90 & 32.38 & 3.97 & 0.006 \\
\hline
\end{tabular}


A

- Death No Death

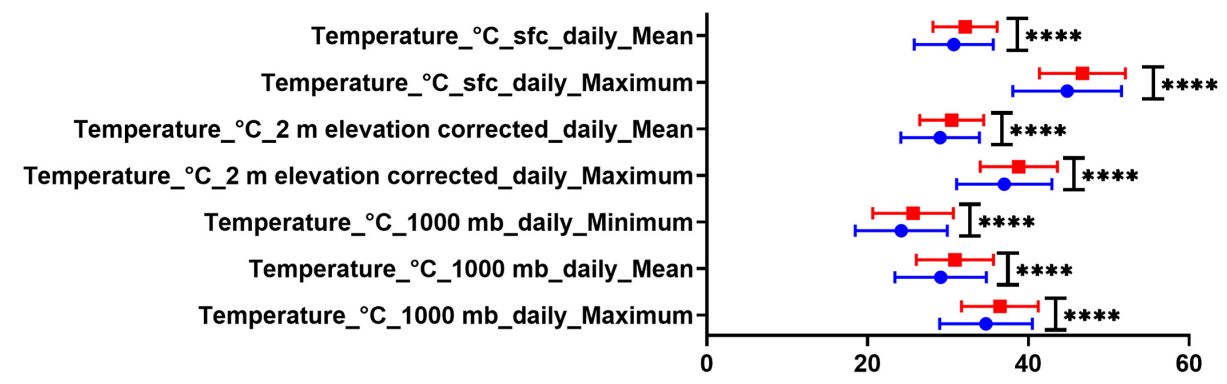

B

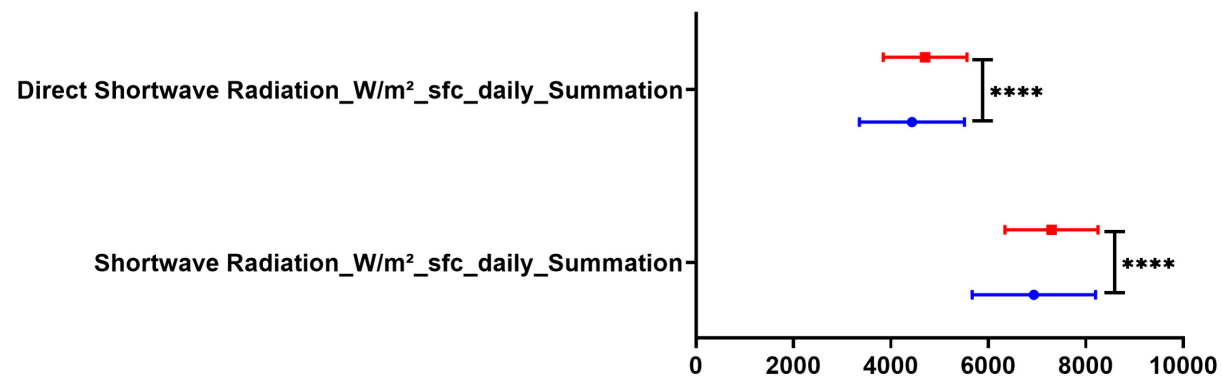

C

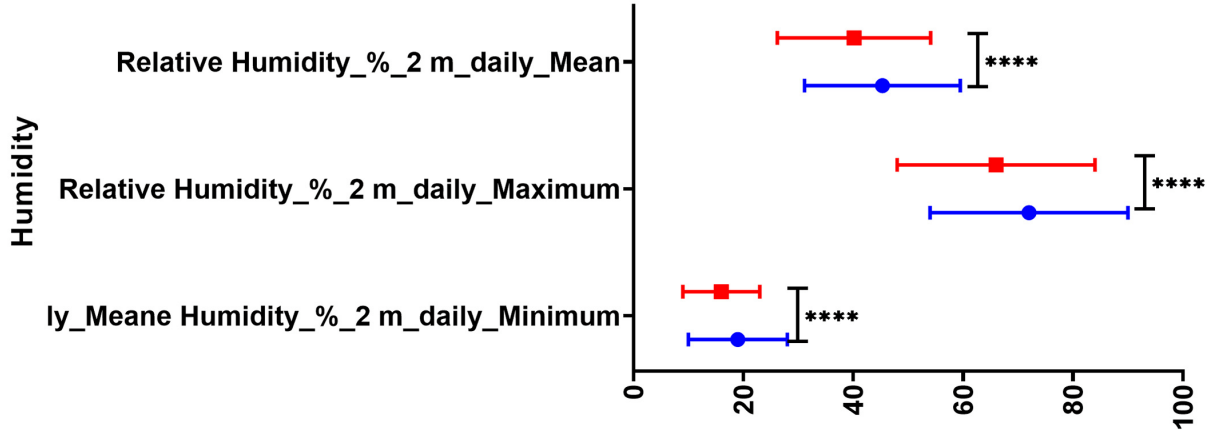

FIGURE 1 | Difference in day admission weather variables in Dubai City between COVID-19 patients with death and those who were discharged in terms of (A) temperature, (B) humidity, and (C) radiation.

$\left({ }^{\circ} \mathrm{C}\right)$ at $2 \mathrm{~m}$ elevation was the most profound factor that is statically different between patients with different COVID-19 severity levels as shown in Table 2. Patients who had severe and critical case of the disease and those who needed ICU were admitted on days with higher temperatures $(23.4 \pm 3.77,23.57$ \pm 3.59 , and $23.14 \pm 3.74, p=0.02$ ). Those who showed a mild to moderate course were admitted in days with lower temperatures $(20.28 \pm 4.07)$ compared to the rest $(23.51 \pm 3.69)$.
Differences in Daily Admission Temperature Affect the Clinical Outcomes in Patients Who Have No Risk Factors to Develop Severe COVID-19

To delineate whether the difference in the clinical outcomes based on daily temperature was different among patients with risk factors to develop severe COVID-19 or not, we divided the patients into those with such risk factors [old age, diabetes 
TABLE 4 | Correlation between the significant weather variables (temperature, humidity, and radiation) on the day of admission with patients' clinical and laboratory parameters.

\begin{tabular}{|c|c|c|c|c|c|c|c|c|c|c|c|c|}
\hline \multirow[t]{2}{*}{ Variables } & \multicolumn{3}{|c|}{$\begin{array}{l}\text { Temperature } \\
{ }^{\circ} \mathrm{C} 2 \mathrm{~m} \\
\text { elevation } \\
\text { corrected } \\
\text { daily mean }\end{array}$} & \multirow{2}{*}{\multicolumn{2}{|c|}{ 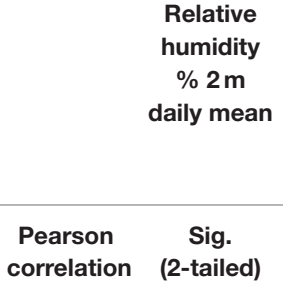 }} & \multirow[b]{2}{*}{$\mathbf{N}$} & \multirow{2}{*}{\multicolumn{2}{|c|}{$\begin{array}{c}\text { Direct } \\
\text { shortwave } \\
\text { radiation } \\
\text { W/m } \mathrm{m}^{2} \text { sfc } \\
\text { daily } \\
\text { summation } \\
\text { Sig. } \\
\text { on } \\
\text { (2-tailed) }\end{array}$}} & \multirow[b]{2}{*}{$\mathbf{N}$} & \multirow{2}{*}{\multicolumn{2}{|c|}{$\begin{array}{c}\begin{array}{c}\text { Temperature } \\
{ }^{\circ} \text { C sfc } \\
\text { daily mean }\end{array} \\
\text { Sig. } \\
\text { (2-tailed) }\end{array}$}} & \multirow[b]{2}{*}{$\mathbf{N}$} \\
\hline & $\begin{array}{l}\text { Pearson } \\
\text { correlation }\end{array}$ & $\begin{array}{c}\text { Sig. } \\
\text { (2-tailed) }\end{array}$ & $\mathbf{N}$ & & & & & & & & & \\
\hline CRP & $0.258^{\star \star}$ & 0 & 429 & $-0.203^{\star \star}$ & 0 & 429 & $0.125^{\star \star}$ & 0.01 & 429 & $0.260^{\star \star}$ & 0 & 429 \\
\hline Neutrophil count & $0.205^{\star \star}$ & 0 & 433 & $-0.175^{\star \star}$ & 0 & 433 & 0.044 & 0.356 & 433 & $0.194^{\star \star}$ & 0 & 433 \\
\hline Age at diagnosis & $0.134^{\star \star}$ & 0.005 & 433 & -0.066 & 0.168 & 433 & $0.146^{\star \star}$ & 0.002 & 433 & $0.148^{\star \star}$ & 0.002 & 433 \\
\hline WCC & $0.131^{\star \star}$ & 0.006 & 433 & $-0.130^{\star \star}$ & 0.007 & 433 & 0.035 & 0.464 & 433 & $0.126^{\star \star}$ & 0.009 & 433 \\
\hline AST & $0.128^{\star \star}$ & 0.008 & 433 & -0.038 & 0.43 & 433 & 0.052 & 0.281 & 433 & $0.137^{\star \star}$ & 0.004 & 433 \\
\hline ALP & $0.111^{\star}$ & 0.021 & 433 & -0.09 & 0.06 & 433 & 0.064 & 0.186 & 433 & $0.112^{\star}$ & 0.019 & 433 \\
\hline $\begin{array}{l}\text { Duration of illness, } \\
\text { days (symptom } \\
\text { onset to } \\
\text { admission) }\end{array}$ & 0.058 & 0.225 & 433 & $-0.099^{\star}$ & 0.04 & 433 & 0.084 & 0.082 & 433 & 0.054 & 0.259 & 433 \\
\hline Lymphocyte count & $-0.104^{\star}$ & 0.031 & 433 & 0.081 & 0.093 & 433 & -0.061 & 0.203 & 433 & $-0.118^{\star}$ & 0.014 & 433 \\
\hline eGFR & $-0.144^{\star \star}$ & 0.003 & 432 & 0.066 & 0.173 & 432 & $-0.128^{\star \star}$ & 0.008 & 432 & $-0.152^{\star \star}$ & 0.002 & 432 \\
\hline $\mathrm{Hb}$ & $-0.162^{\star \star}$ & 0.001 & 433 & $0.120^{*}$ & 0.012 & 433 & $-0.107^{\star}$ & 0.026 & 433 & $-0.161^{\star \star}$ & 0.001 & 433 \\
\hline $\mathrm{Na}$ & $-0.170^{\star *}$ & 0 & 433 & 0.07 & 0.143 & 433 & $-0.119^{\star}$ & 0.013 & 433 & $-0.177^{\star \star}$ & 0 & 433 \\
\hline Albumin & $-0.267^{\star \star}$ & 0 & 432 & $0.258^{\star \star}$ & 0 & 432 & $-0.166^{\star \star}$ & 0.001 & 432 & $-0.263^{\star \star}$ & 0 & 432 \\
\hline
\end{tabular}

${ }^{\star \star}$ Correlation is significant at the 0.01 level (two-tailed).

${ }^{*}$ Correlation is significant at the 0.05 level (two-tailed).

mellitus (DM), hypertension (HTN), cardiovascular disease (CVD), or chronic lung disease] and those without. Differences in daily admission temperature affect the clinical outcomes in patients who have no risk factors to develop severe COVID19, while patients with such risk factors showed no significant difference in the clinical outcomes as shown in Tables 3A-E. This showed that the effect of weather on the clinical outcomes is important in those who have no risk as such risks can affect the outcome regardless of the weather parameters.

\section{The Clinical Outcomes (Death and Organ Failure) of COVID-19 Were Significantly Dependent on the Day-of-Admission Temperature and Relative Humidity}

The next step was to compare the weather parameters of the day of admission between different COVID-19 patients who developed outcomes and complications (like death, acute cardiac injury, acute kidney injury, acute liver injury, acidosis, and septic shock) and those who did not develop such complications.

\section{Death}

There were significant statistical differences in the mean of temperature, relative humidity, shortwave radiation, and direct shortwave radiation between the group of COVID-19 patients who died and the group of COVID-19 patients who survived. COVID-19 patients admitted in days with higher temperatures, higher solar radiation, and less humidity were at higher risk of death, as shown in Figure 1.

COVID-19 patients who died due to the disease were admitted on days with less relative humidity $(40.15 \pm 13.93 \% 2 \mathrm{~m}$ daily mean) compared to those who survived $(45.31 \pm 14.17 \%$ $2 \mathrm{~m}$ daily mean, $p=0.005)$. Also, COVID-19 patients who died due to the disease were admitted on days with higher temperature $\left(32.12 \pm 3.97^{\circ} \mathrm{C}\right.$ sfc daily mean $)$ compared to those who survived $\left(30.71 \pm 4.91^{\circ} \mathrm{C}\right.$ sfc daily mean, $\left.p=0.024\right)$ as shown in Table 3A. COVID-19 patients were divided according to mortality and were compared in terms of day-of-admission weather parameters.

\section{Acute Cardiac Injury}

COVID-19 patients who developed acute cardiac injury were admitted on days with higher temperature $\left(30.77 \pm 3.89^{\circ} \mathrm{C} 2 \mathrm{~m}\right.$ elevation corrected daily mean) compared to patients without cardiac injury $\left(28.81 \pm 4.93^{\circ} \mathrm{C} 2 \mathrm{~m}\right.$ elevation corrected daily mean, $p \leq 0.005$ ) as shown in Table 3B. On the other hand, COVID-19 patients who developed acute cardiac injury were admitted on days with less relative humidity $(41.94 \pm 2.99 \% 2 \mathrm{~m}$ daily mean) compared to the rest of the patients $(45.19 \pm 14.53 \%$ $2 \mathrm{~m}$ daily mean, $p=0.048)$.

\section{Acute Kidney Injury}

COVID-19 patients who showed acute kidney injury were admitted on days with higher temperature $\left(30.42 \pm 3.97^{\circ} \mathrm{C} 2 \mathrm{~m}\right.$ elevation corrected daily mean) compared to patients with intact 
kidney $\left(28.95 \pm 4.92^{\circ} \mathrm{C} 2 \mathrm{~m}\right.$ elevation corrected daily mean, $p=$ 0.01 ) as shown in Table $3 \mathrm{C}$.

\section{Acute Liver Injury}

COVID-19 patients who showed acute liver injury were admitted on days with higher temperature $\left(30.78 \pm 3.89^{\circ} \mathrm{C} 2 \mathrm{~m}\right.$ elevation corrected daily mean) compared to patients with intact liver $\left(28.88 \pm 4.90^{\circ} \mathrm{C} 2 \mathrm{~m}\right.$ elevation corrected daily mean, $\left.p=0.001\right)$ as shown in Table 3D.

\section{Acidosis}

COVID-19 patients who end up with acidosis were admitted on days with higher temperature $\left(30.62 \pm 4.07^{\circ} \mathrm{C} 2 \mathrm{~m}\right.$ elevation corrected daily mean) compared to patients who did not develop acidosis $\left(28.90 \pm 4.89^{\circ} \mathrm{C} 2 \mathrm{~m}\right.$ elevation corrected daily mean, $p=$ 0.003 ) as shown in Table 3E.

\section{Septic Shock}

Interestingly, COVID-19 patients with septic shock were admitted on days with higher temperature $\left(30.73 \pm 3.96^{\circ} \mathrm{C} 2 \mathrm{~m}\right.$ elevation corrected daily mean) compared to patients without septic shock $\left(28.96 \pm 4.88^{\circ} \mathrm{C} 2 \mathrm{~m}\right.$ elevation corrected daily mean, $p=0.005$ ), as shown in Table 3F. Again, COVID-19 patients who developed septic shock were admitted on days with less relative humidity ( $40.63 \pm 13.32 \% 2 \mathrm{~m}$ daily mean) compared to the rest of the patients $(45.20 \pm 14.322 \mathrm{~m}$ daily mean, $p=0.014)$.

\section{Higher Temperature, Less Humidity, and More Radiation on Admission Dates Were Associated With Specific Laboratory Markers}

The next step was to find the correlation between the three weather measurements that were found to be significantly different between the group of COVID-19 that had death as an outcome and the group that survived COVID-19; namely, the weather parameters were high temperature, less humidity, and more radiation. The weather parameters were correlated with patients' clinical and laboratory parameters. The results showed that higher temperature, less humidity, and more radiation on admissions dates were associated with higher CRP, neutrophil count, age at diagnosis, WCC, AST, and ALP but lower lymphocyte count, eGFR, $\mathrm{Hb}, \mathrm{Na}$, and albumin, as shown in Table 4.

\section{DISCUSSION}

High temperatures create a substantial health burden (21) and pose significant public health concerns worldwide, like increased premature deaths attributable to either heat or cold in selected countries (22), but such a burden is not always associated with extreme (high or low) temperatures due to the complexity of weather-related health effects (23). Using the spatial synoptic classification, which uses the combined effect of meteorological factors rather than temperature only for assessing the weather effects on health, is more appropriate to delineate the link between weather and health-related issues (24). For that reason, we explored the different meteorological factors on the outcome of COVID-19 patients in our locally recruited cohort.

Our results showed that in our cohort, COVID-19 patients who were admitted in days with higher temperature, higher solar radiation, and less humidity had a higher chance to develop severe and critical COVID-19, to need ICU admission, and to die than those who were admitted in days with lower temperature and higher relative humidity.

Our results showed also that higher temperature and less humidity were associated with devastating consequences of COVID-19 like acute cardiac, liver and kidney injuries, increased acidosis, and septic shock. All these can explain the positive correlation between higher temperature and less humidity with increased mortality on our cohort. In confirmation to such association, our finding of higher temperature, higher solar radiation, and less humidity association with higher deaths can be linked to the association with markers known to be associated with poor prognosis in COVID-19 patients like higher CRP, neutrophil count, WCC, AST, and ALP and lower lymphocyte count, eGFR, $\mathrm{Hb}, \mathrm{Na}$, and albumin.

But it is not easy to prove causality in such an observational study where many confounding factors might play different roles in the clinical outcomes (6), and that is why we looked for the effect of known risk factors among our cohort to measure the real effect of weather on the outcomes. Interestingly, we found that the effect of weather was more obvious in patients who have no history of risk factors to develop severe COVID-19 as such patients with risk factors showed no difference in their outcomes in different weather conditions. This might indicate the possible direct effect of weather on the consequences of the COVID-19 course. This finding goes with the earlier findings where temperature variation and humidity were found to be important factors affecting COVID-19 mortality (25). Recently, researchers found that there is an observed decrease in COVID19 severity with higher outside temperature, which was explained by restoration of impaired mucosal barrier function due to dry air (6). Substantial community outbreaks of COVID-19 were found to show some preferred latitude, temperature, and humidity measurements, same as other seasonal respiratory virus (26). Climatic factors were thought to affect COVID-19 incidence and severity and can be used in preventive and public health actions against upcoming outbreaks of the disease (27).

So, one can postulate that our finding of more severe COVID19 with lower relative humidity and higher temperature is due to impaired mucosal defense that aids the virus in its infection and propagation. Effective humidity of inhaled air can modulate hydration of the respiratory epithelium that boosts mucosal immunity, so lower relative humidity and higher temperature can actually decrease mucosal hydration and impair it in return (28).

It was shown earlier that the rate of cases presenting daily was inversely associated with daily temperature; such a rate was decreased on days when the temperature was above $52^{\circ} \mathrm{F}$, 5 days earlier (29), as for every $1^{\circ} \mathrm{C}$ increase in temperature, daily new cases of COVID-19 were reduced by 3.08\% (30). So, in our case, the higher temperature might decrease the daily reported cases, but the cases that were admitted despite 
unfavorable weather might be exposed to a higher dose of the virus with closer contact, which can explain the worse course outcome.

The relation between high temperature and mortality in the general population was documented in some reports as it was associated with increased mortality risk, particularly in females and adults aged 20-59 years (31). High air temperature in early summer was associated with increased mortality compared with that in late summer (32), where apparent temperature appeared to be the most critical predictor of heat-related mortality for all-cause mortality (33). On the other hand, the effects of cold on all-cause mortality were highest among people over 75 years old, mainly due to myocardial infarction, ischemic heart diseases, and respiratory diseases (34). Older adults with medical and psychiatric conditions without home heat are most at risk of death due to hypothermia (35).

Patients with chronic diseases may have impaired thermoregulatory ability (36). SARS-CoV-2 is known to increase the hypothalamic-thermoregulatory set, which adversely impacts the outcomes and mortality in patients with COVID-19 (37). Short-term exposure to weather-related stimuli like heat is associated with increased glucocorticoid level that serves as a physiological mechanism promoting fitness during inclement weather, but this, if extended, might have an adverse effect (38). Hot weather in disturbed thermoregulatory conditions induced by SARS-CoV-2 might aggravate mortality.

Heat stress was shown to increase a more significant percentage of neutrophils and a lesser percentage of lymphocytes in animals (39). In humans, heat stress conditions were shown to reduce leukocyte levels, and immunoglobulin concentration can weaken the immune system (40). Elevated ambient temperatures can affect the cardiocirculatory and hormonal systems, resulting in changes in neutrophil and monocyte cell trafficking (41). The neutrophil-to-lymphocyte ratio, interleukin (IL)-6, IL-1 $\beta$, and CRP were higher in persons exposed more frequently to heat per month, which might predispose to systemic inflammation (42). Long-term heat exposure was found to enhance chemokines to recruitment neutrophils to the lungs, leading to an increased risk of respiratory illnesses (43). However, those recruited neutrophils might have impaired phagocytosis and reactive oxygen species (ROS) production under severely high temperatures, leading to a higher occurrence of infections during hot weather (44). Our finding of a correlation between higher temperature and lower eGFR and old age during a presentation can be explained by the fact that older age in men and women exposed to short-term

\section{REFERENCES}

1. Gupta S, Raghuwanshi GS, Chanda A. Effect of weather on COVID-19 spread in the US: a prediction model for India in 2020. Sci Total Environ. (2020) 728:138860. doi: 10.1016/j.scitotenv.2020.138860

2. Adedokun KA, Olarinmoye AO, Mustapha JO, Kamorudeen RT. A close look at the biology of SARS-CoV-2, and the potential influence of weather conditions and seasons on COVID-19 case spread. Infect Dis Poverty. (2020) 9:77. doi: 10.1186/s40249-020-00688-1 ambient temperature was significantly associated with kidney injury biomarkers (45).

In conclusion, our study highlighted the importance of taking weather-related variables into account to understand the dynamics of mortality or clinical outcomes in COVID-19 patients in countries with hot climates like the UAE. The effect of hot stress might weaken the immune system and unleash an inflammatory response that makes some people, especially those with comorbidities or those who are older, more susceptible to infections or to develop aggressive inflammation that ends up with complications and mortality.

\section{DATA AVAILABILITY STATEMENT}

The original contributions generated for this study are included in the article/supplementary material, further inquiries can be directed to the corresponding author/s.

\section{ETHICS STATEMENT}

The studies involving human participants were reviewed and approved by Ministry of Health and Prevention (MOHAP) Research Ethics Committee number (MOHAP/DXB-REC/MMM/NO.44/2020).

\section{CONSENT FOR PUBLICATION}

All authors have agreed to the publication and to be accountable for all aspects of the work in ensuring that questions related to the accuracy or integrity of any part of the work are appropriately investigated and resolved.

\section{AUTHOR CONTRIBUTIONS}

All authors listed have made a substantial, direct and intellectual contribution to the work, and approved it for publication.

\section{ACKNOWLEDGMENTS}

We would like to thank all our patients for their patience. Also, we highly appreciate our colleagues and staff for their tremendous hard work during this crisis. Besides, we extend our gratitude to all staff at the Research Ethics Committee and the Information Technology Department for their help.
3. Raina SK, Kumar R, Bhota S, Gupta G, Kumar D, Chauhan R, et al. Does temperature and humidity influence the spread of Covid19?: a preliminary report. J Family Med Prim Care. (2020) 9:18114. doi: 10.4103/jfmpc.jfmpc_494_20

4. Gardner EG, Kelton D, Poljak Z, Van Kerkhove M, von Dobschuetz S, Greer AL. A case-crossover analysis of the impact of weather on primary cases of Middle East respiratory syndrome. BMC Infect Dis. (2019) 19:113. doi: 10.1186/s12879-019$3729-5$ 
5. Leclercq I, Batéjat C, Burguière AM, Manuguerra JC. Heat inactivation of the Middle East respiratory syndrome coronavirus. Influenza Other Respir Viruses. (2014) 8:585-6. doi: 10.1111/irv.12261

6. Kifer D, Bugada D, Villar-Garcia J, Gudelj I, Menni C, Sudre C, et al. Effects of environmental factors on severity and mortality of COVID-19. medRxiv [Preprint]. (2020). doi: 10.1101/2020.07.11.20147157

7. Sagripanti JL, Lytle CD. Estimated Inactivation of Coronaviruses by Solar Radiation With Special Reference to COVID-19. Photochem Photobiol. (2020) 96:731-7. doi: 10.1111/php.13293

8. Shi P, Dong Y, Yan H, Zhao C, Li X, Liu W, et al. Impact of temperature on the dynamics of the COVID-19 outbreak in China. Sci Total Environ. (2020) 728:138890. doi: 10.1016/j.scitotenv.2020.138890

9. Adhikari A, Yin J. Short-term effects of ambient ozone, $\operatorname{PM}(2.5$,$) and$ meteorological factors on COVID-19 confirmed cases and deaths in Queens, New York. Int J Environ Res Public Health. (2020) 17(11). doi: 10.3390/ijerph17114047

10. Tosepu R, Gunawan J, Effendy DS, Ahmad OAI, Lestari H, Bahar H, et al. Correlation between weather and Covid-19 pandemic in Jakarta, Indonesia. Sci Total Environ. (2020) 725:138436. doi: 10.1016/j.scitotenv.2020. 138436

11. Sahin M. Impact of weather on COVID-19 pandemic in Turkey. Sci Total Environ. (2020) 728:138810. doi: 10.1016/j.scitotenv.2020. 138810

12. Bashir MF, Ma B, Bilal, Komal B, Bashir MA, Tan D, et al. Correlation between climate indicators and COVID-19 pandemic in New York, USA. Sci Total Environ. (2020) 728:138835. doi: 10.1016/j.scitotenv.2020. 138835

13. Zoran MA, Savastru RS, Savastru DM, Tautan MN. Assessing the relationship between surface levels of PM2.5 and PM10 particulate matter impact on COVID-19 in Milan, Italy. Sci Total Environ. (2020) 738:139825. doi: 10.1016/j.scitotenv.2020.139825

14. Qi H, Xiao S, Shi R, Ward MP, Chen Y, Tu W, et al. COVID19 transmission in Mainland China is associated with temperature and humidity: a time-series analysis. Sci Total Environ. (2020) 728:138778. doi: 10.1016/j.scitotenv.2020.138778

15. Malki Z, Atlam ES, Hassanien AE, Dagnew G, Elhosseini MA, Gad I. Association between weather data and COVID-19 pandemic predicting mortality rate: machine learning approaches. Chaos Solitons Fractals. (2020) 138:110137. doi: 10.1016/j.chaos.2020.110137

16. Sharma AK, Balyan P. Air pollution and COVID-19: is the connect worth its weight? Indian J Public Health. (2020) 64:S132-4. doi: 10.4103/ijph.IJPH_466_20

17. Copat C, Cristaldi A, Fiore M, Grasso A, Zuccarello P, Signorelli SS, et al. The role of air pollution (PM and $\mathrm{NO}(2)$ ) in COVID19 spread and lethality: a systematic review. Environ Res. (2020) 191:110129. doi: 10.1016/j.envres.2020.110129

18. Bukhari Q, Massaro JM, D’Agostino RB, Sr., Khan S. Effects of weather on coronavirus pandemic. Int J Environ Res Public Health. (2020) 17:5399. doi: 10.3390/ijerph17155399

19. Yao Y, Pan J, Liu Z, Meng X, Wang W, Kan H, et al. No association of COVID-19 transmission with temperature or UV radiation in Chinese cities. Eur Respir J. (2020) 55:2000517. doi: 10.1183/13993003.00517-2020

20. Iqbal N, Fareed Z, Shahzad F, He X, Shahzad U, Lina M. The nexus between COVID-19, temperature and exchange rate in Wuhan city: new findings from partial and multiple wavelet coherence. Sci Total Environ. (2020) 729:138916. doi: 10.1016/j.scitotenv.2020.138916

21. Guo Y, Gasparrini A, Armstrong BG, Tawatsupa B, Tobias A, Lavigne E, et al. Heat Wave and mortality: a multicountry, multicommunity study. Environ Health Perspect. (2017) 125:087006. doi: 10.1289/EHP1026

22. Gasparrini A, Guo Y, Hashizume M, Lavigne E, Zanobetti A, Schwartz J, et al. Mortality risk attributable to high and low ambient temperature: a multicountry observational study. Lancet. (2015) 386:369-75. doi: 10.1016/S0140-6736(14)62114-0

23. Psistaki K, Paschalidou AK, McGregor G. Weather patterns and allcause mortality in England, UK. Int J Biometeorol. (2020) 64:12336. doi: $10.1007 / \mathrm{s} 00484-019-01803-0$

24. Fonseca-Rodríguez O, Lundevaller EH, Sheridan SC, Schumann B. Association between weather types based on the spatial synoptic classification and all-cause mortality in Sweden, 1991-2014. Int J Environ Res Public Health. (2019) 16:1696. doi: 10.3390/ijerph16101696

25. Ma Y, Zhao Y, Liu J, He X, Wang B, Fu S, et al. Effects of temperature variation and humidity on the death of COVID-19 in Wuhan, China. Sci Total Environ. (2020) 724:138226. doi: 10.1016/j.scitotenv.2020.138226

26. Sajadi MM, Habibzadeh P, Vintzileos A, Shokouhi S, MirallesWilhelm F, Amoroso A. Temperature, humidity, and latitude analysis to estimate potential spread and seasonality of coronavirus disease 2019 (COVID-19). JAMA Netw Open. (2020) 3:e2011834. doi: 10.1001/jamanetworkopen.2020.11834

27. Cacho PM, Hernández JL, López-Hoyos M, Martínez-Taboada VM. Can climatic factors explain the differences in COVID-19 incidence and severity across the Spanish regions?: an ecological study. Environ Health. (2020) 19:106. doi: 10.1186/s12940-020-00660-4

28. Courtney JM, Bax A. Hydrating the respiratory tract: an alternative explanation why masks lower severity of COVID-19 disease. medRxiv [Preprint]. (2020). doi: 10.1101/2020.12.23.20248671

29. Sehra ST, Salciccioli JD, Wiebe DJ, Fundin S, Baker JF. Maximum daily temperature, precipitation, ultraviolet light, and rates of transmission of severe acute respiratory syndrome coronavirus 2 in the United States. Clin Infect Dis. (2020) 71:2482-7. doi: 10.1093/cid/ciaa681

30. Wu Y, Jing W, Liu J, Ma Q, Yuan J, Wang Y, et al. Effects of temperature and humidity on the daily new cases and new deaths of COVID-19 in 166 countries. Sci Total Environ. (2020) 729:139051. doi: 10.1016/j.scitotenv.2020.139051

31. Alam N, Lindeboom W, Begum D, Streatfield PK. The association of weather and mortality in Bangladesh from 1983-2009. Glob Health Action. (2012) 5:53-60. doi: 10.3402/gha.v5i0.19121

32. Luan GJ, Yin P, Wang LJ, You JL, Zhou MG. Association between high air temperature and mortality in summer: A multi-city analysis in China. Zhonghua Liu Xing Bing Xue Za Zhi. (2019) 40:59-63. doi: 10.3760/cma.j.issn.0254-6450.2019.01.012

33. Zhang K, Li Y, Schwartz JD, O'Neill MS. What weather variables are important in predicting heat-related mortality? A new application of statistical learning methods. Environ Res. (2014) 132:350-9. doi: 10.1016/j.envres.2014. 04.004

34. Chen TH, Li X, Zhao J, Zhang K. Impacts of cold weather on all-cause and cause-specific mortality in Texas, 1990-2011. Environ Pollut. (2017) 225:244-51. doi: 10.1016/j.envpol.2017.03.022

35. Lane K, Ito K, Johnson S, Gibson EA, Tang A, Matte T. Burden and risk factors for cold-related illness and death in New York City. Int J Environ Res Public Health. (2018) 15:632. doi: 10.3390/ijerph15040632

36. Nam YH, Bilker WB, Leonard CE, Bell ML, Alexander LM, Hennessy S. Effect of statins on the association between high temperature and all-cause mortality in a socioeconomically disadvantaged population: a cohort study. Sci Rep. (2019) 9:4685. doi: 10.1038/s41598-019-41109-0

37. Suwanwongse K, Shabarek N. Hyperpyrexia in patients with COVID-19. J Med Virol. (2020) 92:2857-62. doi: 10.1002/jmv.26154

38. de Bruijn R, Romero LM. The role of glucocorticoids in the vertebrate response to weather. Gen Comp Endocrinol. (2018) 269:11-32. doi: 10.1016/j.ygcen.2018.07.007

39. Strong RA, Silva EB, Cheng HW, Eicher SD. Acute brief heat stress in late gestation alters neonatal calf innate immune functions. J Dairy Sci. (2015) 98:7771-83. doi: 10.3168/jds.2015-9591

40. Jafari MJ, Pirposhteh EA, Dehghan SF, Khodakarim S, Jafari M. Relationship between heat stress exposure and some immunological parameters among foundry workers. Int $J$ Biometeorol. (2020) 64:853-61. doi: 10.1007/s00484-020-01874-4

41. Niess AM, Fehrenbach E, Lehmann R, Opavsky L, Jesse M, Northoff H, et al. Impact of elevated ambient temperatures on the acute immune response to intensive endurance exercise. Eur J Appl Physiol. (2003) 89:34451. doi: 10.1007/s00421-003-0809-3

42. Watkins ER, Hayes M, Watt P, Renshaw D, Richardson AJ. Extreme occupational heat exposure is associated with elevated haematological and inflammatory markers in Fire Service Instructors. Exp Physiol. (2020) 106:233-43. doi: 10.1113/EP088386

43. Tulapurkar ME, Hasday JD, Singh IS. Prolonged exposure to hyperthermic stress augments neutrophil recruitment to lung during 
the post-exposure recovery period. Int $J$ Hyperthermia. 27:717-25. doi: 10.3109/02656736.2011.601528

(2011)

44. Lecchi C, Rota N, Vitali A, Ceciliani F, Lacetera N. In vitro assessment of the effects of temperature on phagocytosis, reactive oxygen species production and apoptosis in bovine polymorphonuclear cells. Vet Immunol Immunopathol. (2016) 182:89-94. doi: 10.1016/j.vetimm.2016. 10.007

45. Honda T, Manjourides J, Suh $H$. Daily ambient temperature is associated with biomarkers of kidney injury in older Americans. Environ Res. (2019) 179:108790. doi: 10.1016/j.envres.2019. 108790
Conflict of Interest: The authors declare that the research was conducted in the absence of any commercial or financial relationships that could be construed as a potential conflict of interest.

Copyright (๑) 2021 Hachim, Hachim, Naeem, Hannawi, Al Salmi and Hannawi. This is an open-access article distributed under the terms of the Creative Commons Attribution License (CC BY). The use, distribution or reproduction in other forums is permitted, provided the original author(s) and the copyright owner(s) are credited and that the original publication in this journal is cited, in accordance with accepted academic practice. No use, distribution or reproduction is permitted which does not comply with these terms. 